\title{
РАССЛОЕНИЕ АОРТЫ: ПРИЧИНЫ ВОЗНИКНОВЕНИЯ И РЕЗУЛЬТАТЫ ЭКСТРЕННЫХ ОПЕРАЦИЙ
}

\section{AORTIC DISTRIBUTION: CAUSES AND RESULTS OF EMERGENCY OPERATIONS}

\section{Tamer Al Alami \\ Yu. Belov \\ A. Kosenkov}

Summary. Thoracic aortic aneurysms are currently a socially significant problem. This is due to the high mortality rate after the development of complications of this disease - aortic dissection (RA), and the impossibility of conservative treatment. According to modern literature, the incidence of this pathology is about 10.4 per 100,000 population in economically developed countries. In countries with low levels of health care, the frequency may be higher due to the lack of a sufficient level of diagnosis of the population. The causes of this pathology are different. With aneurysms of the ascending aorta (ABA), idiopathic connective tissue dysplasia is most common, and with lesions of the thoracoabdominal department - atherosclerosis. In the studies of a number of authors, a fairly low frequency of coronary vascular damage by the atherosclerotic process in $A B A$ was also noted. At the same time, the frequency of combined aortic valve disease $(A C)$ reaches 5.9 per 100,000 population, i.e. more than half of the cases.

Keywords: mitral regurgitation, Aortic regurgitation, aortic aneurysm, Bentall-deBono operation.

\author{
тамер Аль Алами \\ Аспирант, Первый Московский Государственный \\ Медицинский Университет им. И.М. Сеченова \\ Минздрава России (Сеченовский Университет) \\ r.alami@doctor.com \\ Белов Юрий Владимирович \\ Д.м.н., профессор, Академик РАН, Первый Московский \\ Государственный Медицинский Университет \\ им. И.М. Сеченова (Сеченовский Университет) \\ belov@sechenov.ru \\ Косенков Александр Николаевич \\ Д.м.н., профессор, н.с., Первый Московский \\ Государственный Медицинский Университет \\ им. И.М. Сеченова Минздрава России (Сеченовский \\ Университет) \\ kosenkov@sechenov.ru
}

Аннотация. Аневризмы грудной аорты в настоящее время являются социально-значимой проблемой. Это связано с высокой летальностью после развития осложнений этого заболевания — расслоения аорты (РА), и невозможностью консервативного лечения. По данным современной литературы частота встречаемости данной патологии составляет около 10,4 на 100000 населения в экономически развитых странах. В странах с низким уровнем медицинской помощи частота может быть выше ввиду отсутствия достаточного уровня диагностики населения. Причины развития данной патологии различны. При аневризмах восходящего отдела аорты (ABA) чаще всего встречается идиопатическая дисплазия соединительной ткани, а при поражении торакоабдоминального отдела — атеросклероз. В исследованиях ряда авторов так же отмечена достаточно низкая частота поражения коронарных сосудов атеросклеротическим процессом при ABA. В тоже время частота сочетанного поражения аортального клапана (АК) достигает 5,9 на 100000 населения т.е. более половины случаев.

Ключевые слова: митральная регургитация, Аортальная недостаточность, аневризма аорты, Операция Bentall-deBono.

Последние гистологические исследования указывают на то, что при наличии двустворчатого аортального клапана в восходящем отделе аорты имеются дегенеративные изменения, характеризующиеся фрагментацией эластина и нарушением структуры коллагена и гладкомышечных клеток в стенке аорты [7, с. 588]. В этом же исследовании было проведено сравнение гистологической картины у больных с двустворчатым и трехстворчатым аортальным клапанами. Гистологические изменения были обнаружены у 45\% больных с двустворчатым клапаном и только у 9\% больных с трехстворчатым. При изучении толщины медии у больных 
после операций на сердце обнаружено, что при двустворчатом клапане толщина эластической мембраны меньше, чем при трехстворчатом клапане [8, с. 329].

При операциях на восходящем отделе аорты было установлено, что при двустворчатом аортальном клапане в 8-10 раз чаще развиваются АВА и расширение преимущественно локализуется по передней стенке аорты [9, с. 81]. Даже при нормально функционирующем двухстворчатом АК АВА и РА встречается в $60 \%$ случаев [10, с. 781]. Другими авторами было установлено, что примерно в 80\% случаев патология корня аорты связана с врожденными изменениями в АК [11, с. 49].

До сих пор в литературе нет единого мнения почему при наличии двустворчатого АК развиваются патологические процессы в корне аорты [12, с. 1496]. Одной из причин этому может быть повышенное внимание к этой проблеме врачей терапевтических специальностей, которые рассматривают АК и восходящую аорту как два функционально различных элемента. С точки зрения кардиохирурга это не верно. Корень аорты представляет собой единое анатомо-физиологическое образования, которое начинается от уровня фиброзного кольца аортального клапана и заканчивается сино-тубулярным соединением. Именно в этом заключается наиболее важная особенность понимания данной проблемы.

В единую структуру соединены фиброзные части корня аорты - атрио-вентрикулярный гребень, комиссуральные стержни и фиброзное кольцо сердца. Именно фиброзные элементы корня аорты несут на себе максимальную нагрузку во время сердечного цикла. Во время сокращения сердца происходит повышенное напряжение на фиброзный скелет, а в момент диастолы происходит возращение нагрузки к исходному состоянию [13, с. 325]. Во время сокращения сердца происходит поступление крови в ВА, где имеющийся жидкостной объем распределяется и происходит дальнейшее проталкивание его по сосудистому руслу. При нормально функционирующем АК давление крови на ВА распределяется равномерно без образования турбуленного потока. При развитии двустворчатого АК максимальному давлению подвергается переднебоковая стенка аорты. Исходя из постулата, что при двустворчатому АК чаще встречаются признаки дисплазии соединительной ткани - повышенное давление на изолированный участок аорты чаще приводит к образованию аневризматического расширения. В фазу диастолы за счет имеющегося турбулентного кровотока корень аорты и АК, в частности, испытывают так же перегрузку давлением, это является предпосылками к расширению диаметра фиброзного кольца и развитию аортальной регургитации [14, с. 53]. Кроме наличия врожденного изменения
АК необходимо учитывать особенности физиологии кровотока в восходящей аорте (BА). В норме стенка ВА имеет выраженный эластический компонент, за счет которого происходят процессы «проталкивания» крови по сосудистому руслу [15]. В норме коллаген и эластин являются превалирующими элементами стенки ВА. Однако, при изменении условий окружающей среды, а так же наличии патологических процессов в сосудистом русле возможно развитие структурных нарушений, которые приводят к снижению упругих свойств ВА. К примеру изменения, возникающие с увеличением возраста человека за счет атеросклеротического процесса приводят к снижению дистальной перфузии и увеличению артериального давления на выходе крови из полости левого желудочка.

Одновременно с этим увеличивается нагрузка на сердце и за счет компенсаторного компонента это приводит к увеличению сократимости левого желудочка. Таким образом развивается патологический круг, который невозможно прервать без хирургического вмешательства. Давление в ВА прогрессивно увеличивается, что повреждает даже исходно нормальную стенку аорты, способствует образованию аневризмы.

После обнаружения особенностей, связанных с зависимостью между образованием АВА и возрастом исследователи начали изучать изменения, которые происходят в стенке аорты. Наиболее вероятным маркером «слабости» сосудистой стенки посчитали количество эластина в стенке ВА, поэтому большинство работ построено на оценки этого параметра. В экспериментальных исследованиях было продемонстрировано отсутствие изменения содержания эластина в стенке BA, но в тоже время увеличение других компонентов, например коллагена, которые частично замещали общий объем эластической мембраны сосуда, уменьшая ее упругие свойства

В других исследованиях обнаружили, что аминокислотный состав эластина изменяется с возрастом человека. На протяжении всей жизни человека происходит уменьшение связывающих эластин и коллаген аминокислот, что, по мнению авторов, способствует снижению упругости сосудистой стенки. На фоне этого процесса возможно развитие фрагментации целостной структуры белка у людей с повышенной нагрузкой на стенку грудной аорты или присутствием атеросклеротического процесса. При наличии процессов, способствующих деградации белка эластна в стенке аорты обнаруживаются ферменты семейства металлопротеиназ в высокой концентрации. Вероятно, такие изменения является одним из возможных ступеней апоптоза в организме. Содержание эластина в сухом остатке стенки аорты уменьшается в возрасте старше 20 лет и достигает $33 \%$ 
у здорового человека, а при заболеваниях соединительной ткани процессы развиваются ещё быстрее. Присутствие у больного генетического заболевания соединительной ткани, например синдрома Марфана, стабильность межбелковых структур в эластической мембране снижена на 50\% относительно здорового человека. В других исследованиях продемонстрировано снижение концентрации эластина в стенке аорты при этом заболевании и значительное меньшее количество межструктурных элементов внутри самого белка эластина. Как мы уже указывали ранее, с увеличением возраста больного происходит увеличение коллагена в стенке ВА. При гистологическом анализе было продемонстрировано, что такое увеличение может достигать 30\%. Стоит отметить, что это увеличение происходит не линейно с возрастом человека. Примерно до 45 лет концентрация коллагена остаётся одинаковой, однако далее медленно увеличивается со временем.

При наличии атеросклероза аорты происходит разрушение структуры коллагена IV типа в эластической мембране аорты, однако, этот процесс наблюдается только в области атеросклеротической бляшки. При изучении расслоения аорты на фоне атеросклероза было обнаружено, что в области начала расслоения полностью отсутствует коллагеновая структура, что, вероятно, и приводит к данному осложнению. Если имеет место у больного аневризмой аорты синдрома Марфана, коллагеновая структура в области расширения оставалась не изменой. Однако, межбелковые структуры коллагена в этом случае были обеднены, целостность их нарушена.

Таким образом, по данным литературы можно сделать вывод, что причины развития ABA многогранны, имеют множество предрасполагающих факторов. Однако, основным критерием, который присутствует практически у каждого больного с данным заболеванием является генетическая «слабость» соединительной ткани. В зависимости от степени выраженности генетической мутации аневризмы развиваются или в молодом возрасте, или в пожилом, при наложении на имеющиеся особенности предрасполагающих фактором, например гипертонической болезни. Конечным результатом течения заболевания является расслоение или разрыв аорты.

Общеизвестно, что летальность после острого расслоения аорты типа А (РАA) рассчитывается по схеме $1-2 \%$ в час после начала заболевания в течение первых 48 часов. В связи с этим всех пациентов с РАА необходимо оперировать в срочной порядке. К сожалению, в большинстве случаев РАА диагностируется уже или посмертно, или в сроки, превышающие данные показатели. Поэтому для хирургов наиболее актуальным вопросом становится выбор операции исходя из имеющейся клинической картины.

Острые РАА с клиническими симптомами мальперфузии внутренних органов оперируют в срочном порядке, стабильных больных, чаще всего, в подостром или хроническом периоде. По данным ряда авторов хирургическое лечение в первые часы после эпизода РАА имела место летальность до $27 \%$, в то время как при медикаментозном лечении эти цифры достигали 56\%. Отдаленная выживаемость после хирургического лечения составила $96 \%$ в течение года и $91 \%$ в течение $3-x$ лет, без операции - 89\% и 69\% соответственно. Исходя из этих данных можно сделать вывод о необходимости хирургического лечения любого РАA.

До сих пор не определена тактика лечения в ряде групп больных. Так для больных старше 80 лет, а так же с выраженной мальперфузией и инфарктом головного мозга выживаемость поле хирургического и медикаментозного лечения долгое время считали сопоставимыми. В одном исследовании оценили выбор тактики у больных с неврологическим дефицитом: острое нарушение мозгового кровообращения (ОНМК) и кома. При хирургическом лечении летальность при ОНМК достигала $27 \%$, при коме - 44\%, а при медикаментозном лечении - 76\% и 100\% соответственно. Регрессионный анализ показано, что хирургическое лечение в ранние сроки после начала заболевания увеличивает выживаемость. В другом исследовании сравнивали результаты лечения у молодых больных и лиц старше 70 лет. Авторы продемонстрировали, что возраст не является предиктором большей летальности. В более позднем сообщении показано, что больные старшего возраста, которые перенесли ОНМК и которым не провели хирургического лечения в «холодном» периоде, имели меньшую отдаленную выживаемость без операции, чем с ней. При этом послеоперационная летальность была в 2 раза меньшей, чем при операции в остром периоде ОНМК. Важно отметить, что в такой анализ входят больные, которые пережили первые двое суток после острого РAA.

В случае выполнении операции важным является состоятельность аортального клапана и наличие расслоения корня аорты. При патологической регургитации на АК следует выполнять хирургическую коррекцию данной патологии. «Золотым стандартом» в хирургическом лечении такого состояния на сегодняшний день является операции Bentall-deBono.

В последние годы многие хирурги начали применять клапано-сохраняющие технологии при лечении острого РАA, однако следует отметить, что задачей хирурга при лечении данного состояния является со- 
хранение жизни больного, а не улучшение качества жизни, так же выполнение коронарографии не должно быть рутинным исследованием перед данной операцией, так как на ее проведение требуется время, которое может увеличить дооперационную летальность. Так же объем операции не всегда следует увеличивать до протезирования дуги аорты, в связи с увеличением риском данной процедуры. Только при расслоении аорты 2 типа по DeBakey возможна радикальная коррекция за одну операцию, но зачастую расслоение переходит на торакоабдоминальный отдел аорты, а значит даже расширенная операция не «излечивает» больного.

Реконструкция дуги аорты рекомендована только при ее расширении более 5,5 см, так как это увеличивает риск спонтанного разрыва в этой области.

В ближайшем послеоперационном периоде при коррекции РАА летальность может достигать 30\%, причем одним из факторов риска увеличения летальности является наличие $\mathrm{AH}$, которая требует хирургической коррекции. К другим предикторам развития неудовлетворительных результатов относятся состояния, связанные с мальперфузией органов после случившегося эпизода расслоения. При стабилизации состояния больного, компенсации недостаточности кровообращения по органам и системам в подострый и/или хронический период расслоения возможно добиться снижения летальности более, чем в два раза. В тоже время, для больных с РАA многие авторы предпочитают выполнять «изолированную коррекцию» наиболее измененного участка, это позволяет спасти жизнь больному и минимизирует риски осложнений.

Анализируя отдаленные результаты и причины необходимости повторных вмешательств, большинство авторов обращали особое внимание на состояние АК. Наиболее важно для этих больных было нормализо- вать гемодинамику на уровне корня аорты. Наиболее часто для этого использовали клапаносодержащий кондуит. Такая операция не увеличивала частоту осложнений и позволяла добиться стабильных результатов на протяжении 10 летного периода наблюдения. По результатам авторов до 70\% больных, которым выполнена проксимальная коррекция РАА и которым требовалась повторная операция обращались к хирургу в связи с нарастанием клинической картины сердечной недостаточности на фоне прогрессирования аортальной недостаточности, а не из-за расширения дистальных отделов аорты. В связи с этим, некоторые авторы выполняли коррекцию порока аортального клапана во всех случаях расслоения аорты. но такой подход замедлял тромбоз ложного канала в дистальных отделах аорты в связи с необходимостью постоянной антикоагулянтной терапией.

По данным ряда авторов частота отсутствия тромбоза в ложном просвете расслоения после выполнения операции Bentall-deBono достигала 75-100\% случаев. Расширение дистальных отделов аорты при проксимальной коррекции РАА возникало не более чем у $15 \%$ больных, таким образом увеличение объема операции и риска для больного не оправдано для большинства пациентов.

Исходя их представленных литературных данных считаем, что выбор метода хирургического лечения больных с расслоением аорты следует подбирать индивидуально для каждого больного. При возможности безопасной медикаментозной компенсации органных дисфункций до операции - следует отдавать предпочтение операциям в подостром и хроническом периоде. При жизнеугрожающих состояниях операцию необходимо проводить в экстренном порядке с минимально-радикальным объемом для сохранения жизни больного.

\section{ЛИТЕРАТУРА}

1. Ramanath V S, Oh J K, Sundt T M III, Eagle K A. Acute aortic syndromes and thoracic aortic aneurysm.Mayo Clin Proc. 2009;84(5):465-481, Kuzmik G A, Sang A X, Elefteriades J A. Natural history of thoracic aortic aneurysms. J Vasc Surg. 2012.

2. Белов Ю.В., Комаров Р.Н. Руководство по хирургии торакоабдоминальных аневризм аорты. М.: МИА; 2009.

3. Белов Ю.В., Чарчян Э.Р., Красников М.П. Хирургическая тактика у больных с аневризмой восходящего отдела аорты и ишемической болезнью сердца Кардиология и сердечно-сосудистая хирургия. 2012. Т. 5. № 2. С. 45-48., Agmon Y, Khandheria B K, Meissner I. et al. Is aortic dilatation an atherosclerosisrelated process? Clinical, laboratory, and transesophageal echocardiographic correlates of thoracic aortic dimensions in the population with implications for thoracic aortic aneurysm formation. J Am Coll Cardiol. 2003;42(6):1076-1083, slamoğlu F, Atay Y, Can L. et al. Diagnosis and treatment of concomitant aortic and coronary disease: a retrospective study and brief review. Tex Heart Inst J. 1999.

4. Дземешкевич С.Л., Стивенсон Л.У., Алекси-Месхишвили В.В. Болезни аортального клапана, Москва «Гэотар Медицина» 2004, 328

5. Abbott M.E., Hamilton W.F. Coarctation of the aorta of the adult type. Am. Heart. J. 1928.

6. Ando M., Okita Y., Matsukawa R., et al. Surgery for aortic dissection associated with congenital bicuspid aortic valve. Jpn. J. Thoracic. Cardiovasc. Surg. 1998; 46: 1069-1073, Campbell M. Calcific aortic stenosis and congenital bicuspid aortic valves. Br. Heart. J. 1968; 30; 606-16, Lindsay J. Coarctation of the aorta, bicuspid aortic valve and abnormal ascending aortic wall. Am.J. Cardiol. 1988; 61: 182-4, Paul W., M. Fedak Bicuspid aortic valve syndrome: heterogeneous but 
predictable? European Heart Journal 2008; 29: 432-433, Roberts W.C. The congenitally bicuspid aortic valve: a study of 85 autopsy cases. Am.J. Cardiol 1970; 26: 72-83, Roberts W.C., Morrow A.G., McIntosh C.L. et al. Congenitally bicuspid aortic valve causing severe, pure aortic regurgitation without superimposed infective endocarditis: analysis of 13 patients requiring aortic valve replacement. Am J Cardiol. 1981; 47: 206-209, Roberts W.C. Living with a congenitally bicuspid aortic valve [editorial]. Am J Cardiol. 1989.

7. Белов Ю.В., Комаров Р.Н., Винокуров И.А. Архитектоника, нормальная и патологическая физиология сосудистой стенки Кардиология и сердечно-сосудистая хирургия. 2014. Т. 7. № 6. С. 59-63., Белов Ю.В., Федоров Д.Н., Тааев Б.К., Даабуль А.С. Особенности гистологического строения стенки восходящей аорты при аневризме Кардиология и сердечно-сосудистая хирургия. 2013. Т. 6. № 2. С. 34-36. Mauro de Sa, Y. Moshkovitz, J. Butany and Tirone E. David. Histologic abnormalies of the ascending aorta and pulmonary trunk in patients with bicuspid aortic valve disease: clinical relevans to the Ross procedure. J. Thorac. Cardiovasc. Surg. 1999.

8. Beroukhim R.S., Kruzick T.L., Taylor A.L. et al. Progression of aortic dilation in children with a functionally normal bicuspid aortic valve. Am.J. Cardiol. 2006; 98: 828-830, Nataatmadja M., West M., West J. et al. Abnormal extracellular matrix protein transport associated with increased apoptosis of vascular smooth muscle cells in Marfan syndrome and bicuspid aortic valve thoracic aortic aneurysm. Circulation. 2003.

9. Fedak P.W., Verma S., David T.E. et al. Clinical and pathophysiological implications of a bicuspid aortic valve. Circulation. 2002; 106: 900-4, Ward C. Clinical significance of the bicuspid aortic valve. Heart 2000.

10. Nistri S., Basso C., Marzari C. et al. Frequency of bicuspid aortic valve in young male conscripts by echocardiogram. Am.J. Cardiol. 2005; 96: 718-721, Pachulski R.T., Weinberg A.L., Chan K-L. Aortic aneurysm in patients with functionally normal or minimally stenotic bicuspid aortic valve. Am.J. Cardiol. 1991.

11. Белоусов Ю.В. Охотин И.К. Выбор метода хирургической коррекции врожденного аортального стеноза в зависимости от анатомического варианта. Восстановительная и реконструктивная хирургия. Горький, 1980.

12. Beroukhim R.S., Kruzick T.L., Taylor A.L. et al. Progression of aortic dilation in children with a functionally normal bicuspid aortic valve. Am.J. Cardiol. 2006; 98 : 828-830, Warren A.E., Boyd M.L., 0'Connell C., Dodds L. Dilatation of the ascending aorta in pediatric patients with bicuspid aortic valve: frequency, rate of progression and risk factors. Heart. 2006.

13. Дземешкевич С.Л., Стивенсон Л.У., Алекси — Месхишвили В.В. Болезни аортального клапана. ГЭОТАР-МЕД. Москва. - 2004.

14. Bellhouse B.J. The fluid mechanics of the aortic valve. In: Lonescu M.L. Ross D.N. Woller G.H., eds. Biological tissue in heart valve replacement. London: Butterworth- Heinemann 1972; 23-47, Michael D. Hope, Thomas A. Hope, Alison Meadows K. et al. Bicuspid aortic valve: four-dimensional MR evaluation of ascending aortic systolic flow patterns. Radiology. 2010.

15. Westerhof N, Stergiopulos N, Noble MIM. 2005 Snapshots of hemodynamics: an aidfor clinical research and graduate education. New York, NY: Springer Science. Business Media, Inc.

( ) Тамер Аль Алами ( r.alami@doctor.com ),

Белов Юрий Владимирович ( belov@sechenov.ru ), Косенков Александр Николаевич ( kosenkov@sechenov.ru ).

Журнал «Современная наука: актуальные проблемы теории и практики» 